\title{
Single and Multi-Objective Optimization Algorithms
}

\author{
R. M. Kotb, A. M. Ewais, A. M. Hemeida \\ Electrical Engineering Department \\ Faculty of energy Engineering, Aswan University \\ Aswan, Egypt
}

\begin{abstract}
Hybrid and multi optimization techniques are used extensively for solving optimal power flow problems. In this paper, particle swarm optimization (PSO), is incorporated with grey wolf optimization, (GWO) to form hybrid algorithm called HPSOGWO and using the multi-objective optimization of this algorithm, which called MO-HPSOGWO and comparing them. The HPSOGWO and MO-HP SOGWO are implemented to enhance the optimal power flow solution of ieee-30 bus system. Five objective functions (OPF) optimizing separately by HPSOGWO and simultaneously in a single run by MO-HPSOGWO. The Matlab software is used to solve the system.
\end{abstract}

Keywords - Hybrid and multi optimization techniques, Multi optimization techniques, Single objective functions (OPF).

\section{INTRODUCTION}

Optimization techniques are the best techniques for optimal results for any problem in any field. Traditional optimization techniques are less used due to its key disadvantage: local optimal solution $[1,2]$. The modern optimization techniques are mostly used. It is adjusted by a set of random candidate solutions for a given problem in order to progress them over an adjusted number of steps. Optimization of real world problems requires handling various difficulties such as: multi-objectives [3], uncertainties [4], constraints [5], false global solutions [6], local solutions [7], despite of the advantages of these techniques.

A multi-objective optimization problem consists of a several objective functions more than one function [8]. There is a several non-dominated solutions for a multi-objective problem because of the problems nature [9]. On the other hand, a single objective problem is featured by only one global (best) solution.

There are two basic methods to solve multi-objective optimization problem: a posteriori versus a priori $[3,10]$. For a priori method, we converted a multi-objective optimization problem to a single objective by a set of weights. In this method, an algorithm must be run multiple times to defined the Pareto optimal set, which is considered as the main disadvantages of this method. And some special Pareto optimal fronts cannot be defined with this method [11-13].

At A posterior method, multi-objective formulation of a multi-objective optimization problem is maintained to determine the Pareto optimal set by one run. Moreover, any type of Pareto front can be defined with this method. The main disadvantage of this method is that it has higher computational cost and managing multiple objectives at the same time. There are other popular optimization methods such as: Multi-objective Grey Wolf Optimizer [14], Multiobjective Bee Algorithm [15], Multi-objective Particle Swarm Optimization [16, 17], Multi-objective Bat Algorithm [18], Non-dominated Sorting Genetic Algorithm [19-21].

Hybrid algorithms are a combination between two or more algorithms such as hybrid Particle Swarm Optimization with Gravitational Search Algorithm (PSOGSA) [22,23], and Particle Swarm Optimization with Dragonfly Algorithm (PSODA) [24], and Particle Swarm Optimization with Firefly Algorithm (PSOFA) [25], and Particle Swarm Optimization with Multi Verse Optimizer (PSOMVO) [26].

Regarding to the No-Free Lunch (NFL) theorem, there is no optimization technique for solving all optimization problems [27] making researchers are able to formulate new algorithms or improve it.

\section{PROBLEM FORMULATION}

\section{A. Single objective OPF Problem Formulation}

The mathematical formulation of the OPF problem is presented as a non-linearly constrained optimization problem:

Where:

$$
u=\left[Q_{C}^{T} T C^{T} V_{C}^{T} P_{C}^{T}\right]
$$

$\mathrm{u}=$ the control variables

$Q_{C}=$ reactive power supplied by all shunt reactors

$\mathrm{TC}=$ magnitudes of transformer load tap changer

$V_{G}=$ voltage magnitude at generator buses

$P_{G}=$ active power generated at generator buses

Where:

$$
x=\left[\begin{array}{lll}
V_{L}^{T} \theta^{T} & P_{S G} Q_{G}^{T}
\end{array}\right]
$$

$\mathrm{x}=$ the state variables

$V_{L}^{T}=$ voltage magnitude at load buses

$\theta=$ voltage angles of all buses excluding the slack

bus

$P_{S G}=$ active power generated at the slack bus

$Q_{G}=$ reactive power generated at all generator units

$N_{L}=$ load buses number

$N_{G}=$ generator buses number.

Optimization problem as OPF problem is presented as maximizing or minimizing objective function to be subjected to a set of equality and inequality constraints.

\section{B. Multi-Objective OPF problem Formulation}

Multi-Objective optimization problem consists of several objective functions optimized simultaneously [28, 29]. The Multi-Objective OPF problem is presented as: 
Minimize $\mathrm{f}(\mathrm{x})$ where

$f(x)=\left[f_{1}(x) f_{2}(x) f_{3}(x) f_{4}(x) f_{5}(x)\right]$

Subject to:

$$
x \in X
$$

Where $\mathrm{X}$ is a feasible region:

where

$$
X=\left[x: x \in R^{n}, g_{i}(x) \leq 0, x_{j} \geq 0 \forall_{i}, j\right]
$$

$\mathrm{R}=$ set of real numbers

$g_{i}(x)=$ set of constraints

$\mathrm{x}=$ set of decision variables.

\section{PROBLEM OBJECTIVES}

\section{A. Fuel Cost Minimization}

The economic distribution of a load defined among the different generators of a system, the variable operating costs must be presented as active power generated at each generator in a system. The fuel cost is the essential cost in a thermal or nuclear unit. Then the fuel cost must be presented as active power generated at each generator in a system. Other costs, such as the operation and maintenance costs, can also be presented as the power output. Fixed costs, such as the capital cost, depreciation ..., are not containing in the fuel cost.

A quadratic function of active power generated by each unit in a system approximates the fuel cost curve as:

$F_{1}=\sum_{i=1}^{N_{G}}\left(a_{i}+b_{i} P_{G i}+c_{i} P_{G i}^{2}\right)(R s . / h)$

where:

$P_{G i}$ is the active power generated at an ith generator in a system

$N_{G}$ is the generators number in a system

$a_{i}, b_{i}, c_{i}$ fuel cost coefficients of an ith generator in a system.

\section{B. Emission Minimization}

The function of emission can be aggregated of all types of emission considered, such as $\mathrm{NO}_{x}, \mathrm{SO}_{2}$, thermal emission, etc., As shown in this equation, the amount of emissions is presented as a function of active power generated at each generator in a system, which is the sum of quadratic and exponential functions:

$$
\begin{aligned}
F_{2}=\sum_{i=1}^{N_{G}}\left[10^{-2} *\right. & \left(\alpha_{i}+\beta_{i} P_{G i}+\gamma_{i} P_{G i}^{2}\right) \\
& \left.+\varepsilon_{i} \exp \left(\lambda_{i} P_{G i}\right)\right](t / h)
\end{aligned}
$$

where:

$\alpha_{i}, \beta_{i}, \gamma_{i}, \varepsilon_{i}$ and $\lambda_{i}$ are the emission characteristics coefficients of the ith generator.

\section{Total Real Power Loss Minimization}

The term PL represents the total $I^{2} R$ loss in the transmission lines and transformers of the system. From equation (3) total active power loss equal the sum of generated active power at each generator in a system subtract the sum of an active power at each load bus in a system and the $P_{\text {loss }}$ must be more than zero.

$$
F_{3}=P_{L}=\sum_{i=1}^{N} P_{i}=\sum_{i=1}^{N_{G}} P_{G i}-\sum_{i=1}^{N_{d}} P_{d i}
$$

where

$P_{i}$ real power in each bus:

$P_{d i}$ the demand real power

$N_{d}$ is the load buses number in a network.

$N_{G}$ is the generator buses number.

\section{Reactive Power Transmission Loss Minimization}

Reactive Power Transmission Loss Minimization lead to voltage stability margin (VSM) increasing and enhancing and guarantee good transportation real power from sources to sinks in a network and the $Q_{\text {loss }}$ can be positive or negative value.

$$
F_{4}=Q_{\text {loss }}=\sum_{i=1}^{N} Q_{i}=\Sigma Q_{G i}-\Sigma Q_{d i}
$$

\section{E. Reactive Power Reserve Margin Maximization}

Reactive Power Reserve Margin Maximization leads to minimize reactive power losses and to improve voltage stability and voltage stability under increased load condition or system disturbances. The fast reactive sources are generators, synchronous condensers and FACTs.

$$
p_{5}=\sum_{i=1}^{N_{G}}\left[\frac{Q_{i}^{2}}{Q_{i \max }}\right]
$$

\section{PROBLEM CONSTRAINTS}

\section{A. Equality constraints}

Equality constraints condition can be presented as:

$$
\sum_{i=1}^{N_{G}} P_{G i}-P_{D}-P_{\text {loss }}=0
$$

Where $P_{D}$ and $P_{\text {loss }}$ are demand power and power losses, respectively.

$$
\sum_{i=1}^{N_{G}} Q_{G i}-Q_{D}-Q_{l o s s}=0
$$

\section{B. Inequality constraints}

-Constraints of generation capacity

The generator outputs and bus voltage is restricted by $\min$ and max limits as:

$$
\begin{gathered}
P_{G i}^{\min } \leq P_{G i} \leq P_{G i}^{\max } \\
Q_{G i}^{\min } \leq Q_{G i} \leq Q_{G i}^{\max } \\
Q_{i}^{\text {min }} \leq Q_{i} \leq Q_{i}^{\text {max }} \\
v_{i}^{\text {min }} \leq v_{i} \leq v_{i}^{\text {max }}
\end{gathered}
$$

- Constraints of line flow

This constraints can be presented as:

$$
\left|P_{L f, k}\right| \leq P_{L f, k}^{\max } k=1,2, \ldots \ldots, L
$$


where, $P_{L f, k}$ is the active power flow of line $\mathrm{k} ; P_{L f, k}^{\max }$ is the active power flow high limit of line $k$ and $L$ is the transmission lines number.

\section{THE PROPOSED ALGORITHMS}

The mathematical model for each optimization techniques is explained in this section.

\section{A. Particle Swarm Optimization (PSO)}

The PSO algorithm was firstly invented by Kennedy and Eberhart in 1995 [30,31] and it is based on the imitation of the social behavior of fish, birds and insects and its movement with communication as bird flocking and fish schooling. The word particle indicates, for example, a bee in a colony or a bird in a swarm. Each individual or particle in a swarm be in a organized way by its own intelligence and the collective or group intelligence of the swarm. When one particle finds a good route to food, other particles in a swarm will also be able to follow the good path instantly even if their site is remote from the swarm. This optimization methods based on swarm intelligence are called behaviorally inspired techniques as opposed to the genetic algorithms, which are called evolution-based procedures. It is a population-based technique (a population of particles) and used for optimizing optimization problems. Each particle is supposed to have two characteristics (a position and a velocity). Each particle be around in the search space and can be the best position when evaluated the value of objective function. The particles can be updated a good positions and their velocities based on equations (14) and (15). This approach is learned from swarms behavior to optimize global optimization functions solution and every individual in the swarm is called a particle [32]. These mathematical equations are:

$$
\begin{aligned}
& \quad \omega=\omega_{\max }-k * \frac{\omega_{\max }-\omega_{\min }}{\text { Maxite }} \\
& V_{i j}^{k+1}=\omega * V_{i j}^{k}+c_{1} * r_{1} *\left(\text { Pbest }_{i, j}^{k}-X_{i, j}^{k}\right)+c_{1} * r_{1} * \\
& \left(\text { Gbest }_{j}^{k}-X_{i, j}^{k}\right) \\
& X_{i, j}^{k+1}=X_{i, j}^{k}+V_{i, j}^{k+1}
\end{aligned}
$$

Where, population size is indicated as $\mathrm{N}$ and dimension $\mathrm{D}$ is presented as $\mathrm{X}=\left[\mathrm{X}_{1}, \mathrm{X}_{2}, \ldots, \mathrm{X}_{\mathrm{N}}\right]^{\mathrm{T}}$, where $\mathrm{T}$ indicates the transpose operator. Each particle is presented as $\mathrm{X}_{\mathrm{i}}(\mathrm{i}=1,2$, $\ldots, \mathrm{N})$ is presented as $X i=\left[X_{i, 1}, X_{i, 2}, \ldots, X_{i, D}\right]$. Also, the initial velocity of the population is indicated as $\mathrm{V}=\left[\mathrm{V}_{1}\right.$, $\left.\mathrm{V}_{2}, \ldots, \mathrm{V}_{\mathrm{N}}\right]^{\mathrm{T}}$. Thus, the velocity of each particle in a population $\mathrm{Xi}(\mathrm{i}=1,2, . . \quad, \mathrm{N})$ is presented as $\mathrm{V}_{\mathrm{i}}=$ $\left[V_{i, 1}, V_{i, 2}, \ldots, V_{i, D}\right]$. The index $i$ mutates from 1 to $\mathrm{N}$ whereas the index $j$ mutates from 1 to $\mathrm{D}$.

\section{B. Grey Wolf Optimizer (GWO)}

The GWO algorithm mimes the leadership hierarchy and hunting technique of grey wolves in nature submitted by Mirjalili et al. [33]. Grey wolves are considered to be at the top of food series and they are living in a collection. Four species of grey wolves such as alpha $(\alpha)$, beta $(\beta)$, delta $(\delta)$, and omega $(\omega)$ are simulating the leadership hierarchy and as basic parameters of GWO. As designing GWO according to social hierarchy of wolves, considering the fittest solution as the alpha $(\alpha)$. The second and third best solutions are presented as beta $(\beta)$ and delta $(\delta)$, respectively. The residual of the candidate solutions are supposed to be omega $(\omega)$.

Three basic principles of GWO algorithm, namely hunting, chasing, and tracking for prey, encircling prey, and attacking prey which are considered as the behavior of grey wolves and using for designing GWO. The encircling behavior can be presented as:

$$
\begin{gathered}
\vec{D}=\left|\vec{c} \cdot \vec{X}_{p}(t)-\vec{X}(t)\right| \\
\vec{X}(t+1)=\vec{X}_{p}(t)-\vec{A} \cdot \vec{D}
\end{gathered}
$$

where, $t$ indicates the current iteration, $D, A$, and $C$ indicate coefficient vectors, $X_{p}$ is the prey position vector, and $X$ denotes the grey wolf position vector. The vectors $A$ and $C$ are determined as :

$$
\begin{gathered}
\vec{A}=2 \vec{a} \cdot \vec{r}_{1}-\vec{a} \\
\vec{C}=2 \cdot \vec{r}_{2}
\end{gathered}
$$

simulating the hunting behavior of grey wolves, assuming that the alpha $(\alpha)$, beta $(\beta)$, and delta $(\delta)$ have better knowledge about the probable site of prey. The hunting behavior can be presented as :

$$
\begin{gathered}
\vec{D}_{\alpha}=\left|\vec{C}_{1} \cdot \vec{X}_{\alpha}-\vec{X}\right|, \vec{D}_{\beta}=\left|\vec{C}_{2} \cdot \vec{X}_{\beta}-\vec{X}\right|, \vec{D}_{\delta} \\
=\left|\vec{C}_{3} \cdot \vec{X}_{\delta}-\vec{X}\right| \\
\vec{X}_{1}=\vec{X}_{\alpha}-\vec{A}_{1} \cdot\left(\vec{D}_{\alpha}\right) \\
\vec{X}_{2}=\vec{X}_{\beta}-\vec{A}_{2} \cdot(20) \\
\vec{X}_{3}=\vec{X}_{\delta}-\vec{A}_{3} \cdot\left(\vec{D}_{\delta}\right) \\
\vec{X}(t+1)=\frac{\vec{X}_{1}+\vec{X}_{2}+\vec{X}_{3}}{3}
\end{gathered}
$$

At $|\mathrm{A}|<1$, the wolves are forced to attack the prey, where $A$ is random value. Searching for prey is the exploration ability and attacking the prey is the exploitation ability. At $|\mathrm{A}|>1$ the wolves are enforced to splay from the prey.

\section{A Newly Hybrid Algorithm}

There are a lot of hybridization techniques for heuristic techniques. According to Talbi [34,35], which can be hybridized two techniques or more for hybridization techniques. HPSOGWO is a combination of PSO and GWO. HPSOGWO combines the best strength of both PSO in exploration and in exploitation stage across the targeted optimum solution by replacing the best Value of PSO with grey wolf position value of GWO. In HPSOGWO, first three agents position is updated in the search space by the equations (25-27) with addition inertia constant $(\beta)$ to control the exploration and exploitation of the grey wolf in the search space. The modified equations are presented as:

$$
\begin{aligned}
& \vec{D}_{\alpha}=\left|\vec{C}_{1} \cdot \vec{X}_{\alpha}-\omega * \vec{X}\right| \\
& \vec{D}_{\beta}=\left|\vec{C}_{2} \cdot \vec{X}_{\beta}-\omega * \vec{X}\right| \\
& \vec{D}_{\delta}=\left|\vec{C}_{3} \cdot \vec{X}_{\delta}-\omega * \vec{X}\right|
\end{aligned}
$$


Where, $\omega$ denotes as inertia weight For combining PSO and GWO techniques, the velocity and updated equation are presented as:

$$
\begin{aligned}
V_{i, j}^{k+1}=\omega * V_{i, j}^{k}+ & c_{1} * r_{1} *\left(X_{1}-X_{i, j}^{k}\right)+c_{2} * r_{2} \\
& *\left(X_{2}-X_{i, j}^{k}\right)+c_{3} * r_{3} \\
& *\left(X_{3}-X_{i, j}^{k}\right) \\
X_{i, j}^{k+1}= & X_{i, j}^{k}+V_{i, j}^{k+1}
\end{aligned}
$$

\section{The basic steps of HPSOGWO}

STEP 1: Create an initial population (agents) or (Grey wolves).

STEP 2: Initialize a,A,C and $\omega$ equations( $13,18,19)$. STEP 3: Fitness evaluation of each agents.

STEP 4: Calculate the position of Grey Wolf. $X_{\alpha}, X_{\beta}, X_{\delta}$ equations(25-27) and (21-23).

STEP 5: Updating velocity and position equations $(28,29)$.

STEP 6: Repeat STEP (2-5) until the stop criteria is reached.

STEP 7: Stop.

\section{Multi-OBJective OptimizATION}

Multi-Objective optimization problem consists of several objective functions to be optimized simultaneously. In these problems, objective functions are in conflicting with each other. For example, in Optimal Power Flow problem (OPF), by minimizing generation fuel cost and consequently active and reactive power losses are maximizing. Main concepts related to Multi-Objective Optimization are [36]:

\section{A. Domination}

In Multi-Objective optimization the domination is used for comparing the solutions as in Fig 1 . If all the X1 solution are not worse than all solution X2 in all objectives, or if all the $\mathrm{X} 1$ solutions are equal to $\mathrm{X} 2$ but only in one case or one dimension $\mathrm{X} 1$ is better than $\mathrm{X} 2$, then it can be said that $\mathrm{X} 1$ will dominate $\mathrm{X}_{2}$ and $\mathrm{X}_{2}$ must be deleted from solutions list. The mathematical expression of the domination part is presented as :

$$
\begin{gathered}
x_{1} \text { dominate } x_{2} \text { if }: \\
f\left(x_{1}\right) \leq f\left(x_{2}\right) \\
f\left(x_{1}\right)<f\left(x_{2}\right)
\end{gathered}
$$

\section{B. Pareto front}

A solution of Multi-Objective optimization problem is a curve not a point but a set of points, which every point in this curve will non-dominate each other. Because if find a point in which one of the target functions is minimum, there is another target function which is not minimum at this point. This curve called Pareto front curve as in Fig 2.

\section{Finding best local guide}

A solution of Multi Objective optimization problem is a set of Pareto optimal solutions not the best point. Pareto optimal solutions obtained in each iteration are stored in an archive (Repository) and this archive is updated in each iteration to make the domination points deleted. All the Pareto optimal solutions in the archive are equally good.

\section{D. A Multi-OBJECTIVE HybrID ALGORITHM (MO-HPSOGWO)}

In order to implement multi-objective optimization by HPSOGWO we combine two new components. The components are similar to MOPSO [17,18] and MOGWO [21].The first one is the repository (archive), which is responsible for storing non-dominated Pareto optimal solutions obtained so far and there is a maximum number of solutions for the repository. The second component is a leader selection designing that assists to select alpha, beta and delta solutions as the leader of the hunting process from the repository.

The MO-HPSOGWO algorithm inherits all the characteristics of HPSOGWO, which means that there are the same exploration and exploitation abilities in two algorithms. The basic difference that MO-HPSOGWO design about the repository (archive), which the solution is a set of non-dominated solutions not three best solutions as HPSOGWO algorithm.

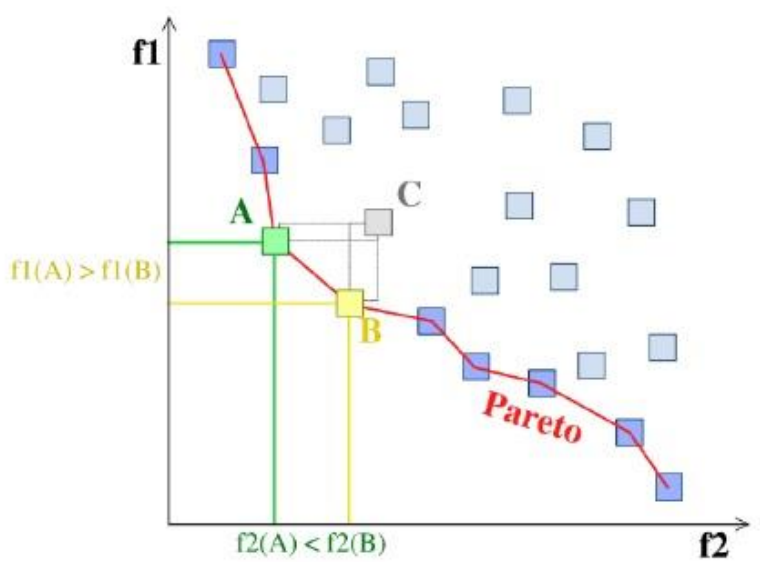

Fig.1. Pareto optimum [37]

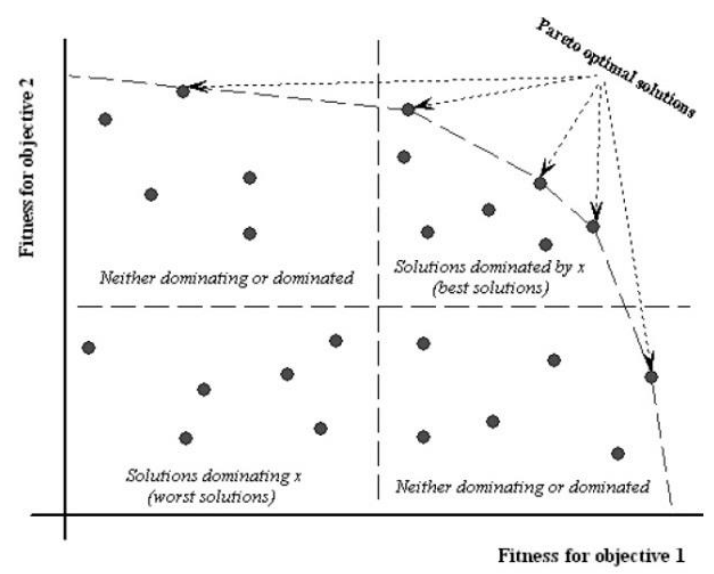

Fig.2. Concept of pareto optimality [38] 


\section{PSEUDO CODE OF THE MO-HPSOGWO ALGORITHM}

Create an initial population $\mathrm{X}_{\mathrm{i}}(\mathrm{i}=1,2, \ldots, \mathrm{n})$

Initialize a, A,C and $\omega$ equations $(13,18,19)$

Fitness evaluation of each agents

Find the non-dominated points and initialized the repository with them

$X_{\alpha}=$ SelectLeader(rep)

Exclude $\alpha$ from the repository tentatively to avoid selecting the same leader

$X_{\beta}=$ SelectLeader(rep)

Exclude $\beta$ from the repository tentatively to avoid selecting the same leader

$X_{\delta}=$ SelectLeader(rep)

Add back alpha and beta to the repository

$\mathrm{t}=1$;

for $(\mathrm{t}=1$ : Max iterations)

for each agents

Update the position of the current search agent by equations(25-27) and (21-23)

Update the velocity and position by equations $(28,29)$ end

\section{for}

Update a, A, C and $\omega$

Fitness evaluation of each agents

Find the non-dominated points

Update the repository

If the repository is complete

Run the grid mechanism to delete one of the current repository points

Add the new point to the repository

end if

$X_{\alpha}=$ SelectLeader(rep)

Exclude $\alpha$ from the repository tentatively to avoid selecting the same leader

$X_{\beta}=$ SelectLeader(rep)

Exclude $\beta$ from the repository tentatively to avoid selecting the same leader

$X_{\delta}=$ SelectLeader(rep)

Add back alpha and beta to the repository

$\mathrm{t}=\mathrm{t}+1$

return rep

\section{RESULTS AND DISCUSSION}

shown in Fig.9 The total active power demands is 283.4 MW and Total reactive power demands is 126.2 MVAR. Five objective functions (OPF) are individually optimized as a single objective function in optimization process by using HPSOGWO and are optimized simultaneously by using MO-HPSOGWO, which are :

$F_{1}$ Fuel Cost Minimization

$F_{2}$ Emission Minimization

$F_{3}$ Total Active Power Loss Minimization

$F_{4}$ Reactive Power Transmission Loss Minimization

$F_{5}$ Reactive Power Reserve Margin Maximization.
From TABLE III : in single optimization process there is one best (global) solution for each function without attention to the value of the other four functions. For example, when minimizing of generation fuel cost this leads to active and reactive power losses in a system increasing and vice versa.

From TABLE IV : there are several solutions (nondominated) for five functions (OPF) as in Fig.8, which are optimized simultaneously. And these five functions are conflicting objectives that means when decision optimal solution need to trade off between them. So the decision maker (DM) for selecting a compromise solution based on maximum limits as in TABLE II and minimum limits as in TABLE III.

TABLE I. Load flow analysis of 30 bus system by using NR method

\begin{tabular}{|c|c|c|c|c|}
\hline Bus No. & V(p.u.) & Delta & $\mathrm{P}(\mathrm{MW})$ & Q(MVAR) \\
\hline 1 & 1.050 & 0.0 & 353.099 & -14.98 \\
\hline 2 & 1.038 & -3.705 & 54.28 & 18.011 \\
\hline 3 & 1.011 & -10.514 & -145.380 & 10.748 \\
\hline 4 & 1.019 & -8.316 & -15.700 & 12.855 \\
\hline 5 & 1.091 & -8.667 & 24.280 & 22.753 \\
\hline 6 & 1.091 & -10.383 & 24.000 & 20.940 \\
\hline 7 & 1.006 & -9.554 & -45.600 & -10.900 \\
\hline 8 & 1.016 & -6.857 & -15.200 & -1.600 \\
\hline 9 & 1.048 & -9.932 & 0.000 & 0.000 \\
\hline 10 & 1.031 & -11.672 & -11.600 & -2.000 \\
\hline 11 & 1.023 & -5.708 & -4.800 & -1.200 \\
\hline 12 & 1.065 & -11.212 & -22.400 & -7.500 \\
\hline 13 & 1.015 & -7.994 & 0.000 & -0.000 \\
\hline 14 & 1.047 & -12.088 & -12.400 & -1.600 \\
\hline 15 & 1.039 & -12.108 & -16.400 & -2.500 \\
\hline 16 & 1.043 & -11.666 & -7.000 & -1.800 \\
\hline 17 & 1.030 & -11.876 & -18.000 & -5.800 \\
\hline 18 & 1.024 & -12.654 & -6.400 & -0.900 \\
\hline 19 & 1.019 & -12.787 & -19.000 & -3.400 \\
\hline 20 & 1.021 & -12.565 & -4.400 & -0.700 \\
\hline 21 & 1.019 & -12.132 & -35.000 & -11.200 \\
\hline 22 & 1.020 & -12.119 & -0.000 & 0.000 \\
\hline 23 & 1.023 & -12.434 & -6.400 & -1.600 \\
\hline 24 & 1.009 & -12.527 & -17.400 & -6.700 \\
\hline 25 & 1.010 & -12.469 & -0.000 & -0.000 \\
\hline 26 & 0.993 & -12.894 & -7.000 & -2.300 \\
\hline 27 & 1.020 & -12.163 & 0.000 & 0.000 \\
\hline 28 & 1.012 & -8.478 & 0.000 & 0.000 \\
\hline 29 & 1.000 & -13.401 & -4.800 & -0.900 \\
\hline 30 & 0.989 & -14.290 & -21.200 & -1.900 \\
\hline
\end{tabular}

TABLE II. IEEE 30-bus system individual objective functions before applying optimization technique

\begin{tabular}{|c|c|}
\hline Objective function & Objective value \\
\hline Over all Generation fuel costs & $4205.1(\$ / \mathrm{h})$ \\
\hline Emission index & $2.4681(\mathrm{t} / \mathrm{h})$ \\
\hline Active power transmission loss & $19.579(\mathrm{MW})$ \\
\hline Reactive power transmission loss & $5.827(\mathrm{MVar})$ \\
\hline Reactive power reserve margin & 1.1034 \\
\hline
\end{tabular}


TABLE III. Single objective function values by HPSOGWO.

\begin{tabular}{|c|c|}
\hline Function & Best solution \\
\hline $\mathrm{F}_{1}$ (min of fuel cost) & 739.838 \\
\hline $\mathrm{F}_{2}$ (min of emission) & $2.0486 \mathrm{E}-04$ \\
\hline $\mathrm{F}_{3}($ min of active power loss $)$ & 5.28 \\
\hline $\mathrm{F}_{4}($ min of reactive power loss $)$ & -16.02 \\
\hline $\mathrm{F}_{5}$ (max of reactive power reserve $)$ & $1.17 \mathrm{E}-16$ \\
\hline
\end{tabular}

TABLE IV. Multi-objective functions (OPF) values (non-dominated solutions) by MO-HPSOGWO

\begin{tabular}{|c|c|c|c|c|c|}
\hline NO. & $\begin{array}{c}\mathrm{G}_{\mathrm{FC}} \\
(\mathrm{Rs} / \mathrm{h})\end{array}$ & $\begin{array}{c}\mathrm{E}_{\mathrm{I}} \\
(\mathrm{t} / \mathrm{h})\end{array}$ & $\begin{array}{c}P_{\text {loss }} \\
(M W)\end{array}$ & $\begin{array}{c}Q_{\text {loss }} \\
(\text { MVar })\end{array}$ & $\begin{array}{c}\mathrm{R}_{\mathrm{PRM}} \\
(\mathrm{p} . \mathrm{u})\end{array}$ \\
\hline 1 & 778.892 & 0.0904 & 0.901 & 3.501 & 0.806 \\
\hline 2 & 1628.2 & 1.1583 & 11.581 & -7.867 & 0.213 \\
\hline 3 & 805.713 & 0.1356 & 1.354 & 2.016 & 0.931 \\
\hline 4 & 785.456 & 0.0487 & 0.484 & 10.316 & 1.086 \\
\hline 5 & 976.827 & 0.3706 & 3.704 & -0.181 & 0.711 \\
\hline 6 & 787.768 & 0.0011 & 0.008 & 8.575 & 1.377 \\
\hline 7 & 779.825 & 0.0134 & 0.132 & 10.625 & 1.053 \\
\hline 8 & 1067.4 & 0.4988 & 4.986 & -2.586 & 0.223 \\
\hline 9 & 823.879 & $2.48 \mathrm{E}-04$ & $2.36 \mathrm{E}-04$ & 14.316 & 1.376 \\
\hline 10 & 799.699 & 0.013 & 0.129 & 12.327 & 1.156 \\
\hline 11 & 805.841 & 0.132 & 1.317 & 4.186 & 0.584 \\
\hline 12 & 1054.9 & 0.4445 & 4.442 & -0.419 & 0.663 \\
\hline 13 & 858.655 & 0.1986 & 1.983 & 3.199 & 0.695 \\
\hline 14 & 803.943 & $2.91 \mathrm{E}-04$ & $4.59 \mathrm{E}-04$ & 12.252 & 1.249 \\
\hline 15 & 772.455 & 0.0506 & 0.504 & 5.517 & 1.143 \\
\hline 16 & 938.129 & 0.3297 & 3.295 & 0.830 & 0.630 \\
\hline 17 & 986.147 & 0.3724 & 3.722 & 0.239 & 0.483 \\
\hline 18 & 812.773 & 0.0038 & 0.035 & 10.605 & 1.248 \\
\hline 19 & 1885.6 & 1.449 & 14.494 & -10.689 & 0.142 \\
\hline 20 & 806.641 & $3.05 \mathrm{E}-04$ & $4.44 \mathrm{E}-04$ & 11.896 & 1.573 \\
\hline 21 & 794.102 & 0.0168 & 0.165 & 10.329 & 0.990 \\
\hline 22 & 1360.5 & 0.8531 & 8.528 & -6.064 & 0.312 \\
\hline 23 & 888.950 & 0.2575 & 2.573 & 0.417 & 0.720 \\
\hline 24 & 1332.4 & 0.8148 & 8.145 & -5.114 & 0.423 \\
\hline 25 & 765.056 & 0.0227 & 0.224 & 5.935 & 1.398 \\
\hline 26 & 854.473 & 0.1956 & 1.954 & 3.593 & 0.668 \\
\hline 27 & 858.135 & 0.2078 & 2.076 & 2.245 & 0.558 \\
\hline 28 & 761.863 & 0.0634 & 0.632 & 5.078 & 0.857 \\
\hline 29 & 783.092 & 0.0165 & 0.163 & 10.118 & 1.122 \\
\hline 30 & 819.717 & $4.15 \mathrm{E}-04$ & 0.002 & 11.945 & 1.461 \\
\hline 31 & 786.184 & 0.0061 & 0.059 & 9.857 & 1.398 \\
\hline 32 & 781.588 & 0.0067 & 0.064 & 8.211 & 1.675 \\
\hline 33 & 804.136 & 0.0856 & 0.854 & 7.028 & 0.846 \\
\hline 34 & 1477.9 & 0.9816 & 9.814 & -6.846 & 0.309 \\
\hline 35 & 1992.6 & 1.5829 & 15.826 & -12.984 & 0.258 \\
\hline 36 & 772.595 & 0.0493 & 0.491 & 6.269 & 1.109 \\
\hline 37 & 1008.3 & 0.4093 & 4.090 & 0.227 & 0.703 \\
\hline 38 & 774.865 & 0.0343 & 0.340 & 6.896 & 1.326 \\
\hline 39 & 778.702 & 0.0255 & 0.253 & 9.663 & 1.128 \\
\hline 40 & 773.374 & 0.0544 & 0.542 & 6.308 & 0.843 \\
\hline
\end{tabular}

TABLE V. The standard values used

\begin{tabular}{|c|c|}
\hline Parameters & quantity \\
\hline population size & 100 \\
\hline Repository size (rep) & 100 \\
\hline No. of iterations & 200 \\
\hline
\end{tabular}

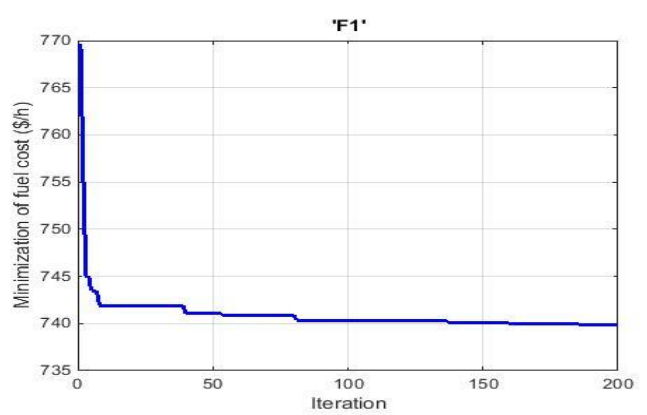

Fig.3. Minimization of generation fuel cost by HPSOGWO

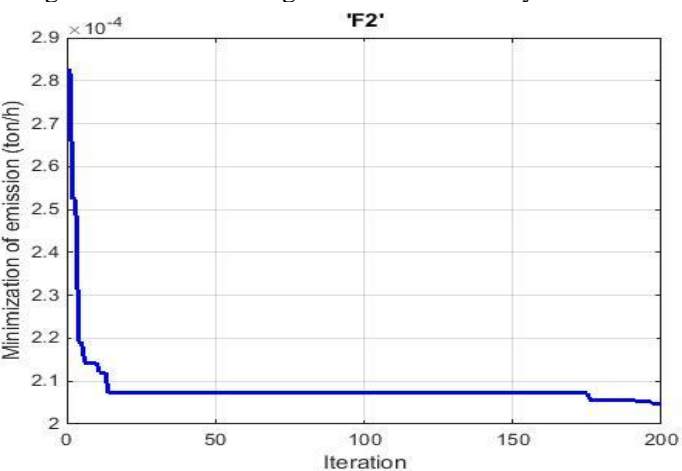

Fig.4. Minimization of emission by HPSOGWO

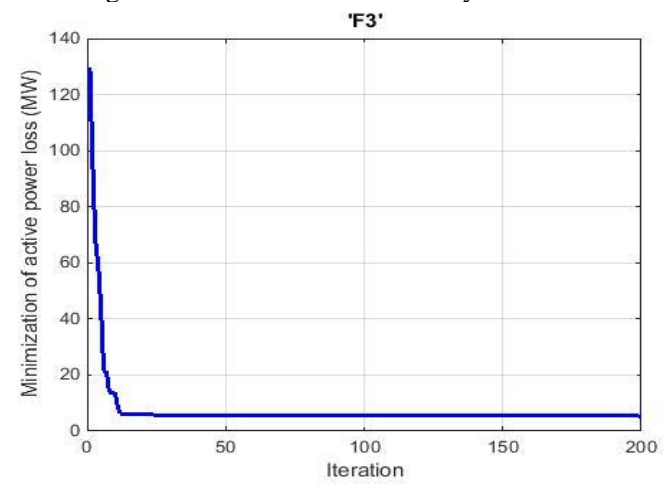

Fig.5. Minimization of active power loss by HPSOGWO

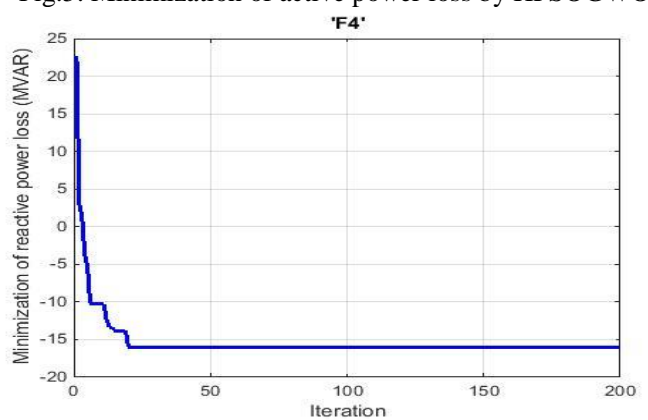

Fig.6. minimization of reactive power loss by HPSOGWO 


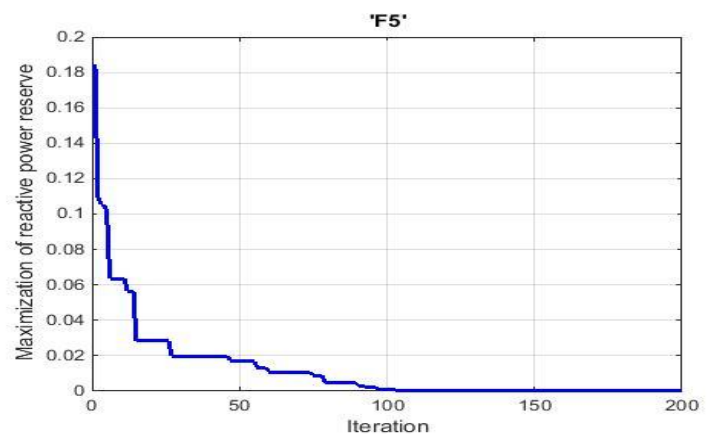

Fig.7. maximization of reactive power reserve margin by HPSOGWO

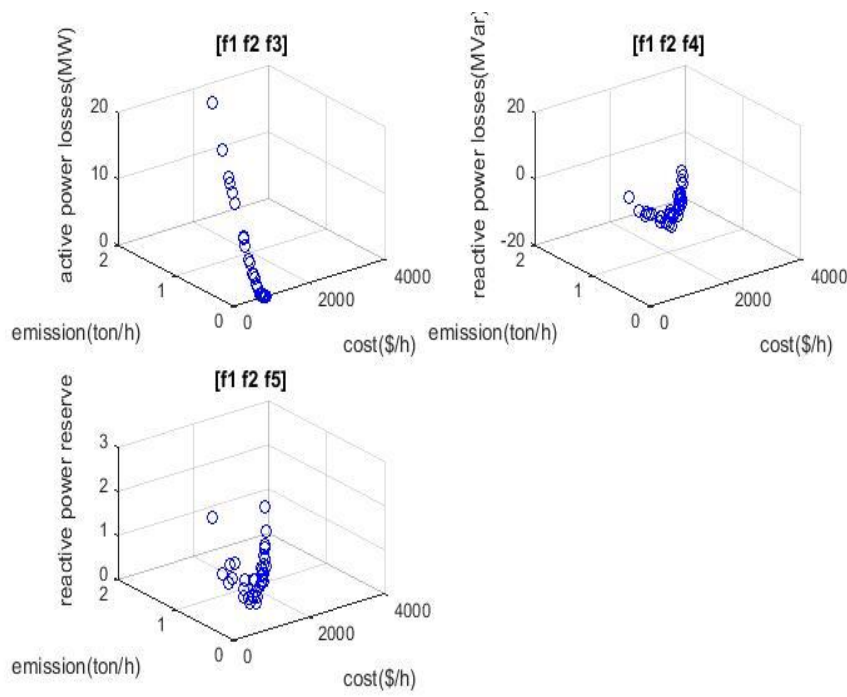

Fig.8. Pareto optimal for five function (OPF) non-dominated solutions by HPSOGWO

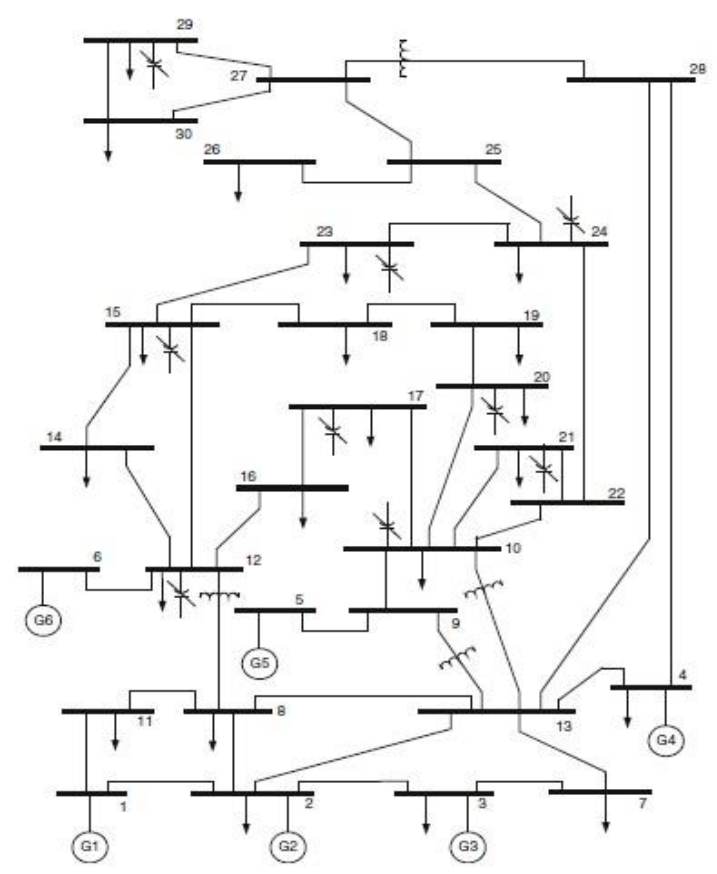

Fig.9. Single-line diagram of IEEE-30 bus test system

\section{CONCLUSION}

Most of the real world problems in many fields science, engineering, economics and logistics are multi-objectives optimization problems, making conflicting objectives. In this paper using two algorithms HPSOGWO and MOHPSOGWO testing through IEEE 30-bus system and optimizing five objective function (OPF). From results MOHPSOGWO is more realistic and efficient than HPSOGWO because single objective function (HPSOGWO) has one global solution without attention to the value of the other four functions as in TABLE III but multi-objective functions (MO-HPSOGWO) has a set of non-dominated solutions and the compromise solution selecting based on decision maker (DM) as in TABLE IV and these five functions can be optimized concurrently. So without using multi-objective optimization in (OPF) only one aspect of the power system has been optimized.

\section{REFERENCES}

[1] C. T. Kelley, "Detection and remediation of stagnation in the Nelder Mead algorithm using a sufficient decrease condition," (1999).

[2] T. P. Vogl, J. Mangis, A. Rigler, W. Zink, D. Alkon, “ Accelerating the convergence of the back-propagation method,". (1988).

[3] R. T. Marler, J. S. Arora, "Survey of multiobjective optimization methods for engineering,". (2004)

[4] H. G. Beyer, B. Sendhoff, "Robust optimization a comprehensive survey," (2007).

[5] C. Coello, "Theoretical and numerical constraint-handling techniques used with evolutionary algorithms: a survey of the state of the art,". (2002)

[6] K. Deb, D. E. Goldberg, "Analyzing deception in trap functions," (1993)

[7] Knowles JD, Watson RA, Corne DW, "Reducing local optima in single objective problems by multi-objective," In: Evolutionary multicriterion optimization, pp.269283, (2001).

[8] Deb K, "Multi-objective optimization using evolutionary algorithms, "Vol.16, Wiley,(2001).

[9] Coello CAC, Lamont GB and David A. Van Veldhuisen, "Evolutionary algorithms for solving multi-objective problems," Springer.

[10] Branke J, Deb K, Dierolf H, Osswald M, “Finding knees in multiobjective optimization," (2004).

[11] Das I, Dennis JE, “Normal boundary intersection: A new method for generating the Pareto surface in nonlinear multicriteria optimization problems," (1998).

[12] Kim IY, De Weck, “Adaptive weighted-sum method for bi-objective optimization: Pareto front generation," (2005).

[13] Messac, A., and Mattson, C. A., "Generating Well-Distibuted Sets of Pareto Points for Engineering Design using Physical Programming, " Optimization and Engineering, Vol. 3, Issue 4, December 2002, pp. 431-450.

[14] Mirjalili S, Saremi S, Mirjalili SM, Coelho L. d. s., "Multi objective grey wolf optimizer: A novel algorithm for multi-criterion optimization, " (2016).

[15] Akbari R, Hedayatzadeh R, Ziarati K, Hassanizadeh B, “A multiobjective artificial bee colony algorithm, " In Swarn and Evolutionary Computation, February 2012..

[16] Coello CAC, Lechuga MS, "MOPSO: A proposal for multiple objective particle swarm optimization," (2002).

[17] Coello CAC, Pulido GT, Lechuga MS, “ Handling multiple objectives with particle swarm optimization," IEEE Transactions on Evolutionary Computation,June 2004. 
[18] Xin-She Yang, "Bat algorithm for multi-objective optimization," ,September 2011,Intenational Journal of Bio-Inspired Computation.

[19] Deb K, Agrawal S, Pratap A, Meyarivan T, "A fast elitist nondominated sorting genetic algorithm for multi-objective optimization: NSGA-II," (2000).

[20] Deb K, Goel T, "Controlled elitist nondominated sorting genetic algorithms for better convergence," (2001).

[21] Deb K, Pratap A, Agarwal S, Meyarivan T, "A fast and elitist multiobjective genetic algorithm: NSGA-II, ” (2002)

[22] S. Mirjalili and S. Z. M. Hashim, "A new hybrid PSOGSA algorithm for function optimization, " in Proceedings of the International Conference on Computer and Information Application (IC- CIA '10), pp. 374377, Tianjin, China, November (2010).

[23] Hardiansyah, "A Novel Hybrid PSO-GSA Method for Non-convex Economic Dispatch Problems, ” Published Online November (2013) in MECS DOI: 10.5815/ijieeb.2013.05.01.

[24] Trivedi I.N., Jangir P., Kumar A., Jangir N., Bh- esdadiya R.H., Totlani R., "A Novel Hybrid PSO-DA Algorithm for Global Numerical Optimization," (2018).

[25] S. Arunachalam, T. AgnesBhomila, and M. Ramesh Babu, "Hybrid Particle Swarm Optimization Algorithm and Firefly Algorithm Based Combined Economic and Emission Dispatch Including Valve Point Effect, ” in July (2015).

[26] Pradeep Jangir, Siddharth A.Parmar, Indrajit N.Trivedi, and R.H. Bhesdadiya, "A novel hybrid Particle Swarm Optimizer with multi verse optimizer for global numerical optimization and Optimal Reactive Power Dispatch problem, " in April (2017).

[27] Wolpert DH, Macready WG, "No Free Lunch theorems for optimization," April,1997, IEEE Transactions on Evolutionary Computation

[28] S.A.H. Soliman, A.H. Mantawy, "Modern Optimization Techniques With Applications in Electric Power Systems, "Energy System Springer, NewYork, (2012).

[29] Wadhwa, C.L., Jain, N.K, “Multiple objective optimal load flow: A new perspective," (1990).

[30] J. Kennedy and R. Eberhart, "Particle swarm optimization," in Proc. IEEE Int. Conf. Neural Networks (ICNN'95), Perth, Australia, IV: 19421948, (1995).

[31] Y. Shi and R. Eberhart, "A modified particle swarm optimizer, "Proceedings of IEEE International Conference on Evolutionary Computation, Anchorage, Alaska, 69-73, (1998).
[32] Peter J.Angeline, “Evolutionary optimization versus particle swarm optimization:Philosophy and performance differences," In: Proceedings of 7th Annual Conference Evolutionary Programming, San Diego, pp. 601610,March,1998.

[33] S. Mirjalili, S. M. Mirjalili, and A. Lewis, "Grey wolf optimizer, "Advances in Engineering Software, vol. 69, pp. 4661, (2014).

[34] Narinder Singh and S. B. Singh, "Hybrid Algorithm of Particle Swarm Optimization and Grey Wolf Optimizer for Improving Convergence Performance, " Department of Mathematics, Punjabi University, Patiala, Punjab 147002, India, (2017).

[35] Talbi, "A taxonomy of hybrid metaheuristics, " Journal of Heuristics, vol. 8, no. 5, pp. 541564, (2002).

[36] Seyed Saeed Hosseini, Sajad Ahmad Hamidi, Motahar Mansuri and Ali Ghoddosian, "Multi Objective Particle Swarm Optimization (MOPSO) for Size and Shape Optimization of 2D Truss Structures, "pp.9-14, (2015).

[37] Carlos A. Santos Silva, "Multi-objective Optimization, " MIT Portugal.

[38] Ankit Yadav and Dr.Sanjay K.Jain, "MULTIOBJECTIVE OPTIMAL POWER FLOW, ” July 2010, Thapar university,Patiala. 